\section{Regards sur l'économie allemande}

Bulletin économique du CIRAC

$66 \mid 2004$

Varia

\title{
Recherche universitaire et intégration européenne
}

\author{
Marie-Hélène Pautrat
}

\section{OpenEdition}

\section{Journals}

Édition électronique

URL : http://journals.openedition.org/rea/3853

DOI : 10.4000/rea.3853

ISBN : 978-2-8218-0828-7

ISSN : 1965-0787

Éditeur

CIRAC

Édition imprimée

Date de publication : 1 mai 2004

Pagination : 19-24

ISSN : 1156-8992

Référence électronique

Marie-Hélène Pautrat, «Recherche universitaire et intégration européenne », Regards sur l'économie allemande [En ligne], 66 | mai 2004, mis en ligne le 12 octobre 2009, consulté le 01 mai 2019. URL : http://journals.openedition.org/rea/3853 ; DOI : 10.4000/rea.3853 


\title{
Recherche universitaire et intégration européenne
}

\author{
Marie-Hélène Pautrat
}

Acteurs essentiels de la recherche en Allemagne, les établissements d'enseignement supérieur sont replacés au cœur de la grande offensive du gouvernement fédéral qui a fait de 2004 l'année de l'innovation. Le débat politique s'est recentré sur l'impact du système scientifique dans la compétitivité du pays. II adhère ainsi pleinement à l'ambition européenne de renforcer le rôle de la science dans le développement de l'Europe. La recherche est explicitement reconnue comme point d'ancrage de toute innovation. Universités d'élite, réseaux d'excellence, européanisation des universités sont autant de stratégies que choisit l'Allemagne pour réaliser les objectifs de Lisbonne et de Barcelone d'une société de la connaissance qui passe par l'intégration des capacités scientifiques au sein d'un Espace Européen de la Recherche (EER). Mais l'effort doit être poursuivi : au plan purement comptable, l'Allemagne est encore loin de l'engagement d'élever ses dépenses nationales de R\&D de 2,48\% aujourd'hui à $3 \%$ du PIB. Et si la qualité de la recherche universitaire allemande demeure à ce jour reconnue en dépit d'un sous-financement préoccupant, la communauté scientifique ne manque pas de déplorer que certains aspects structurants du système handicapent, à ses yeux, son maintien dans la compétition mondiale.

En termes de dépenses de R\&D, les établissements de l'enseignement supérieur représentaient en 2000 le deuxième acteur du système de recherche, derrière les entreprises et devant les organismes publics et privés de recherche extra-universitaire. Leurs dépenses se sont élevées à 8,0 milliards $€$ dont près d'un tiers $(2,6$ milliards $€$ ) relevait de financements sur projet (Drittmittel). Le financement de l'activité de recherche des universités a été assuré à $87 \%$ (7 milliards $€$ ) par les autorités publiques, et en premier lieu les Länder, dont une part de la dotation de base revient à la recherche. Quant au Bund, il participe au financement de la construction de bâtiments, au financement des programmes spéciaux consacrés à "l'amélioration des capacités de recherche universitaire » et, indirectement, par les crédits attribués par la Deutsche Forschungsgessellschaft DfG dont près de $80 \%$ reviennent aux universitaires. Enfin, Bund et Länder octroient aux établissements des financements sur projets.

Dépenses de R\&D en RFA et part de l'enseignement supérieur (en millions $€$ )

\begin{tabular}{|lrrr|}
\hline & $\mathbf{1 9 9 5}$ & $\mathbf{1 9 9 7}$ & $\mathbf{2 0 0 0}$ \\
Enseignement supérieur & & & \\
Financement & 7378 & 7677 & 8034 \\
- par entreprises & 605 & 747 & 909 \\
- par pouvoirs publics & 6694 & 6798 & 6987 \\
- par institutions privées à but non lucratif & - & - & -- \\
- par étranger & 78 & 131 & 139 \\
Dépense intérieure brute de R\&D & & & \\
Financement & 40658 & 42859 & 49822 \\
- par entreprises & 24852 & 26300 & 32656 \\
- par pouvoirs publics & 14960 & 15386 & 15928 \\
- par institutions privées à but non lucratif & 104 & 141 & 209 \\
- par étranger & 741 & 1032 & 1030 \\
Dépense brute de R\&D en \% du PIB & $\mathbf{2 , 2 6}$ & $\mathbf{2 , 3 4}$ & $\mathbf{2 , 4 5}$ \\
\hline
\end{tabular}

Source: Faktenbericht Forschung 2002 (p. 350) 
Progression de la R\&D des entreprises dans les universités

Universités : au cœur du système de recherche etd'innovation

Une qualité reconnue de formation et de recherche

La constitution d'une Europe de la recherche
Les entreprises participent elles aussi à l'activité de recherche des universités (910 millions $€$ soit $11,3 \%$ ). Elles effectuent d'ailleurs une part, en nette progression, de leur recherche dans les laboratoires universitaires : les dépenses de recherche universitaire financées par les entreprises ont plus que doublé depuis 1991 alors que, dans le même temps, les dépenses de R\&D des entreprises progressaient de $38,7 \%$ seulement. En termes de personnel de recherche, les organismes universitaires employaient 101500 personnes en équivalent temps plein en 1999 , soit $21,8 \%$ de l'ensemble du personnel de recherche. Ce ratio demeure inférieur à la moyenne européenne (34\% selon la Commission européenne).

L'enseignement supérieur allemand est caractérisé par différents types d'établissements. On restreindra ici volontairement son champ aux universités, car ce sont elles qui pratiquent l'essentiel de la recherche dans l'enseignement supérieur. En dehors même de tout critère quantitatif, c'est en effet l'interaction étroite entre les différentes activités des universités qui les place au centre du système de recherche et d'innovation. Le modèle humboldtien, qui continue de marquer de son empreinte l'université allemande, suppose en effet une unité très forte entre enseignement et recherche. La recherche constitue de ce fait une activité centrale, menée de manière complémentaire avec les grands organismes extra-universitaires. Par ailleurs, les universités sont responsables de la formation des chercheurs qui irriguent l'ensemble des centres de recherche publics et privés en Allemagne... et à l'étranger. L'Allemagne n'échappe pas non plus au phénomène préoccupant de « fuite des cerveaux ». Les établissements universitaires contribuent à la formation d'un personnel très qualifié pour tous les domaines connexes de l'innovation. Enfin, une des missions essentielles de l'université demeure le transfert des connaissances et des résultats des recherches menées en son sein. Les établissements allemands ont noué de longue date des coopérations nombreuses et variées avec les milieux économiques et leur environnement local. Ce dernier point continue néanmoins d'être considéré comme le maillon faible du système, en dépit d'infrastructures et d'institutions reconnues (comme la fondation Steinbeis) et d'une dynamique relancée depuis la fin des années 90 (par exemple, le programme Exist pour la création d'entreprises innovantes à partir des universités).

Malgré la virulence des critiques auxquelles elles sont soumises dans le débat public, les universités allemandes continuent de bénéficier d'une large reconnaissance internationale pour le potentiel de recherche qu'elles proposent dans une palette disciplinaire particulièrement étendue, ainsi que par le nombre d'établissements et de facultés susceptibles de se hisser à un niveau mondial. Pour discutés qu'ils soient, plusieurs indicateurs viennent confirmer la reconnaissance de qualité qu'on attribue traditionnellement aux établissements universitaires allemands : reconnaissance des post-docs allemands dans les laboratoires étrangers, nombre de Prix Nobel issus des établissements allemands, nombre de boursiers allemands dans les universités à l'étranger, nombre de publications, etc.

D'un point de vue qualitatif, l'université allemande semble donc bien armée pour prendre la place que la Commission européenne l'invite à occuper dans le projet de "société de la connaissance ». Lors des Conseils européens de Lisbonne et de Barcelone, les pays membres se sont en effet engagés à faire des systèmes européens d'enseignement et de recherche une « référence de qualité mondiale » d'ici 2010. L'objectif est de mieux intégrer les capacités scientifiques et technologiques de chaque pays-membre. L'articulation des efforts nationaux a pour objectif de dégager une véritable "plus-value " au sein d'un Espace Européen de la Recherche. Les universités sont ainsi doublement concernées par l'agenda européen : à la fois dans leur mission d'enseignement et dans leur activité de recherche. Ainsi que l'a formulé la Commission en février 2003, elles sont invitées à accélérer les démarches d'européanisation qui de- 
meurent peut-être plus visibles à l'heure actuelle dans le domaine de l'enseignement (avec la réforme des diplômes) que de la recherche.

A ce titre, la participation des établissements universitaires allemands aux dispositifs européens constitue un indicateur pertinent de leur capacité à s'insérer dans des réseaux et de trouver toute leur place au sein de l'Espace européen de la Recherche (EER). Les programmes communautaires de coopération internationale dans l'enseignement supérieur, comme TEMPUS (coopération universitaire avec les pays de l'ex-URSS, du Sud-Est européen et les pays méditerranéens partenaires), Asia-Link ou Alfa (Amérique latine), mais aussi l'implication dans certaines actions européennes sur l'innovation technologique (par exemple le développement des parcs scientifiques, le soutien à l'exploitation des résultats de la recherche, etc.) témoignent de la réactivité des établissements, de leur ouverture et de leur volonté de rayonnement. Les Programmescadre pluriannuels de R\&D technologique de l'Union européenne (PCRDT) constituent la pièce maîtresse du dispositif européen de soutien à la recherche. Si l'on s'en tient au volume des fonds mobilisés (15 milliards $€$ pour le $5^{\mathrm{e}}$ PCRDT - 1998-2002, 17,5 milliards $€$ pour le $\left.6^{\mathrm{e}}-2002-2006\right)$, les financements engagés n'excèdent pourtant guère $5 \%$ de la dépense intérieure de R\&D des pays membres. Mais la participation au PCRD est de plus en plus considérée comme un critère essentiel, car structurant, du positionnement au sein de l'EER : il permet d'évaluer la réactivité des laboratoires nationaux face à de nouveaux enjeux scientifiques et sociaux et leur capacité à travailler au sein de réseaux transfrontaliers. Avec un taux de participation en forte progression (18,1 \% pour le $5^{\mathrm{e}}$ PCRDT contre $15,8 \%$ pour le $4^{\mathrm{e}}$ ), l'Allemagne conforte progressivement son intégration dans le paysage de la recherche européenne.

Sur un total de 11327 projets enregistrés, l'Allemagne est en effet présente dans 5 622, soit dans près d'un projet sur deux (49,6\%). A titre de comparaison, le rapport de l'Observatoire des Sciences et des Techniques indique que la France, avec 4495 projets (39,7\%), figure en troisième position derrière l'Allemagne et le Royaume-Uni (46,0\%). La KoWi (Koordinationsstelle EG für Wissenschafts-organisationen) a publié en 2003 un bilan de la participation des établissements allemands de l'enseignement supérieur dans le cadre du $5^{\mathrm{e}}$ PCRDT. 15408 participations de laboratoires académiques ont été enregistrées, soit 3 partenaires universitaires par projet sur une moyenne de 7 . Sur ce total, les universités allemandes ne sont pas en reste : elles se sont investies dans plus de 5000 projets, menés en coopération avec des partenaires représentant au total 71 pays. La Grande-Bretagne a constitué le partenaire scientifique privilégié des universités allemandes au cours de ce cinquième PCRDT, avec 781 collaborations (soit plus de $23 \%$ ). Elle est suivie de l'Italie $(11,2 \%)$ et de la France (10,03\%). Mais il faut noter également que 10,8\% des collaborations menées par les universités allemandes l'ont été avec des établissements de pays-candidats à l'Union européenne.

Si on se réfère au nombre de participations au $5^{\mathrm{e}}$ PCRDT enregistrées, trois Länder se situent en bonne position. Ce sont d'abord le Bade-Wurttemberg, avec 20 établissements universitaires représentés (dont l'université de Stuttgart, l'université technique de Karlsruhe et l'université de Tübingen) et la Rhénanie du Nord-Westphalie avec 27 universités (en tête, la RWTH d'Aix-La Chapelle, les universités de Bonn et de Dortmund). Ces deux Länder bénéficient, il est vrai, d'une forte concentration d'établissements du supérieur sur leur territoire. La Bavière suit en troisième position avec 10 universités, dont I'Université technique de Munich, I'Université Ludwig-Maximilians de Munich et l'université d'Erlangen. Côté thématique, l'investissement des laboratoires universitaires allemands est plus marqué dans les domaines «Société de l'information conviviale » (664 participations), "Qualité de la vie et gestion des ressources du vivant » (589), «Energie, environnement et développement durable » (383), « Croissance compétitive et durable » (348) et « Confirmer le rôle international de la recherche communautaire » (258).
L'intégration européenne de la recherche universitaire

Les universités allemandes ont tiré profit du $5^{\mathrm{e}}$ PCRDT,...

... dans 3 Länder surtout 
L'Allemagne bénéficie largement du $6^{\mathrm{e}}$ PCRDT

Quels enjeux pour les établissements universitaires?

Des établissements préparés aux exigences du PCRD

Et dotés d'outils de gestion des projets
Le $6^{e}$ PCRDT étant en cours, il est trop tôt pour envisager une analyse différenciée de l'implication de chacun des acteurs de la recherche et développement. Le ministère fédéral de l'enseignement et de la recherche (BMBF) a néanmoins fait part début février 2004 d'une forte participation allemande dans plus de $80 \%$ des projets, qu'il interprète comme le juste retour des efforts importants de conseil et de communication mis en œuvre : organisation de réunions d'information pour la communauté scientifique, création de sites Internet consacrés au $6^{\mathrm{e}}$ PCRD (dont le portail : www.rp6.de), ambition de renforcer dans le cadre de l'élargissement la dynamique de coopération scientifique avec les pays candidats. Cet investissement participe de la volonté clairement exprimée d'augmenter les participations de l'Allemagne. Dans les secteurs porteurs des nanotechnologies, des biotechnologies, des TIC, de l'espace, de l'énergie, des transports et de l'environnement, l'écart se creuse d'ailleurs entre l'Allemagne et les autres pays de l'Union : un cinquième du financement consacré à ces domaines (total : 5 milliards $€$ ) revient d'ores et déjà aux laboratoires allemands.

Mais les objectifs de ce $6^{\mathrm{e}}$ PCRDT ne sont pas sans poser quelques défis aux universités allemandes. Ce dispositif pose en effet les premiers jalons dans la structuration de l'EER et d'une politique scientifique et technologique commune. Il est donc utile de s'intéresser à l'impact sur les universités des principes-clés de ce nouveau PCRD : la concentration du soutien financier sur quelques domaines ciblés, à progrès rapide, l'évaluation de l'impact des recherches en termes d'innovation sociale et économique, et l'invitation à créer et gérer des structures communes de recherche. Deux types d'instruments sont particulièrement mis en exergue : les réseaux d'excellence et les projets intégrés, qui tous deux visent à mobiliser une masse critique de moyens.

Pour les universités allemandes, les choix opérés depuis une dizaine d'années par la plupart des Länder peuvent laisser penser que les établissements sont globalement sensibilisés à ce type d'enjeux. Les politiques régionales visant à renforcer le profil des établissements, en fonction de domaines de spécialisation, se sont en effet multipliées sous la pression des arbitrages budgétaires. Mais en parallèle, la réduction du soutien européen à quelques priorités spécifiques (et à un nombre limité de réseaux) porte en germe le risque de marginaliser des domaines universitaires de recherche innovants ou porteurs à plus ou moins long terme. Très concrètement, le principe de constitution de réseaux va révéler la capacité des établissements scientifiques à s'inscrire dans des consortiums, voire à les coordonner, avec les conséquences qui en découlent : capacité à mobiliser une expertise juridique sur les questions de contrat, de protection de la propriété intellectuelle, aptitude au management de projets et notamment gestion financière, calcul des coûts, etc.

Quelques universités ont d'ailleurs développé ces dernières années des filiales (comme la société Attempto Service $\mathrm{GmbH}$, filiale de l'Université de Tübingen) ou ont vu des structures émerger dans leur giron (par exemple Eurice - European Research and Project Office $\mathrm{GmbH}$, spin off issue de l'université de la Sarre). A l'interface entre milieux universitaires et économiques, ces sociétés proposent une gamme plus ou moins étendue de services. Elles offrent notamment une assistance dans la gestion des projets de recherche et proposent la mise en œuvre et la coordination de coopérations nationales ou transfrontalières dans le cadre d'appels d'offre européens. Enfin, on peut s'attendre à ce que les établissements allemands soient, à l'avenir, mieux armés pour les questions de management de projets. Ils disposent en effet d'un personnel administratif et technique plus nombreux (qu'en France en tout cas) et se dotent progressivement depuis quelques années d'outils de gestion financière, comptables et de management nouveaux. Pourtant, en fonction du type d'université, des disciplines représentées et des choix politiques opérés par les tutelles régionales, tous les établissements n'ont pas, il est vrai, la même capacité à répondre aux enjeux posés par l'européanisation et l'internationalisation de la recherche. 
Dans ce contexte, le chancelier Schröder et sa ministre de l'éducation et de la science, Mme Buhlman, ont engagé début 2004 une vaste initiative destinée à susciter une dynamique de mobilisation autour de l'innovation et du renforcement de la visibilité et de l'attractivité du système scientifique allemand. De son côté, avec ses "Orientations de Weimar » (Weimarer Leitlinien), le SPD s'engageait fortement à faire de l'innovation un enjeu politique majeur. II invitait dès lors les établissements universitaires, en tant que pièce maîtresse du dispositif, à répondre au double défi d'une augmentation du nombre de diplômés du supérieur et de l'excellence. A l'occasion de son discours de politique générale du 25 mars dernier, très attendu par les milieux de la recherche, le chancelier a annoncé la redistribution de 5 milliards $€$ par an (débloqués par la suppression de subventions dans le cadre de l'accès à la propriété) en faveur des établissements d'enseignement supérieur. Par ailleurs, le gouvernement a mis à l'étude la vente (encore hypothétique à ce jour) d'une partie des réserves en or de la banque fédérale dont le produit servirait à la création d'une fondation pour la recherche et le développement.

Mais la mesure phare pour les établissements universitaires reste l'émergence du concept, très fortement médiatisé, d'universités d'élite (Spitzenuniversität). Cette idée n'a cessé de faire l'objet d'interprétations fort divergentes durant le premier trimestre 2004, en particulier entre le Bund et la KMK, la Conférence des ministres de l'éducation et des sciences des Länder. Initialement, la ministre fédérale souhaitait doter 5 établissements, promus « universités d'élite ", d'un financement complémentaire annuel de 50 millions $€$ accordés à chacun pendant 5 ans, dans une logique de création d'établissements " phares » facilement identifiables dans le paysage scientifique mondial (suivant les modèles de Harvard ou Oxford). Arguant qu'impératif d'excellence et précarité financière se combinent mal, les Länder ont défendu a contrario le principe d'une sélection au niveau disciplinaire, par un soutien à une vingtaine de "centres d'excellence » à identifier dans les facultés, et par la mise en place d'écoles doctorales (Graduiertenkollegs) de grande qualité pour garantir une relève scientifique de haut niveau. Ce choix cadre finalement davantage avec la position de la Commission européenne qui préconise une plus forte spécialisation des universités et une évaluation des points forts dans chaque système national, pour globalement atteindre à l'échelon européen un niveau d'excellence.

Finalement, les deux parties semblent avoir trouvé un terrain d'entente, en annonçant à la fin mars l'organisation d'un concours commun répondant à une triple ambition. II devra permettre de distinguer un nombre encore inconnu (une dizaine ? une quinzaine ?) d'établissements, de créer de nouvelles écoles doctorales, et d'identifier des " centres d'excellence ». Ces derniers pourront également intégrer des Fachhochschulen et des UFR, ce dernier élément faisant l'objet d'un compromis entre le niveau des établissements, privilégié jusqu'alors par le Bund, et le niveau disciplinaire. Les lauréats se partageront entre 2006 et 2011 une enveloppe globale annuelle abondée par le Bund et les Länder. Si le Bund s'engage sur un montant annuel de 250 millions $€$, la contribution des Länder n'est pas précisée. Au mieux, elle permettra de doubler l'apport du gouvernement fédéral.

Ces annonces n'auront suffi à lever ni le scepticisme, ni la vigilance des milieux universitaires. Côté financement d'abord: alors que le gouvernement annonce une hausse de $3 \%$ en 2005 du budget du BMBF, la Conférence des recteurs et présidents des établissements d'enseignement supérieur (HRK) rappelle que, au plan purement comptable, l'engagement européen d'une progression à $3 \%$ du PIB des dépenses nationales de R\&D équivaut à des taux de croissance bien supérieurs du budget alloué aux organismes de recherche et aux universités : de l'ordre de $8 \%$ par an. Dans ses " Huit thèses sur la place des universités dans le système scientifique ", publiées en février et suivies peu de temps après d'une déclaration commune des présidents des organismes scientifiques allemands, la HRK insistait une nouvelle fois sur le sous-financement structurel
L'offensive gouvernementale pour l'innovation

Des interprétations divergentes de l'excellence universitaire

Compromis sur une triple ambition

Les « huit thèses » de la HRK 
Revendications récurrentes des milieux universitaires

qui enferme les établissements dans des contraintes de plus en plus inextricables. Cet état de fait n'est pas en cohérence avec le rôle moteur assigné à l'enseignement et la recherche pour l'innovation économique et sociale. Les établissements sont ainsi soumis à des pressions en sens contraire : les effectifs étudiants en hausse, doublés d'un décrochage de l'emploi enseignant et scientifique et d'une dégradation des conditions matérielles d'exercice de leur activité, ne leur permettraient pas de réunir les conditions d'une recherche et d'un enseignement d'excellence. De plus, la focalisation, surtout à moyens constants, sur quelques établissements et réseaux, qu'ils soient d' "élite » ou d'« excellence », risque de s'effectuer au détriment de tous les autres, si le financement de l'ensemble du système universitaire n'est pas garanti.

Acceptant malgré tout le principe d'une compétition sur les moyens dans la politique universitaire et scientifique (mais sur la base d'une évaluation scientifique des disciplines et des chercheurs), la HRK a souhaité rappeler aux tutelles publiques un certains nombre d'autres contraintes qui émaneraient cette fois directement de l'environnement réglementaire : conditions de rémunération et de carrière trop peu attractives et trop indifférenciées, manque d'autonomie budgétaire, procédures de nomination dans les universités jugées non compétitives pour attirer des chercheurs étrangers, freins à la mobilité des étudiants étrangers. II s'agit là de revendications récurrentes de la HRK et des responsables d'organismes scientifiques, auxquelles les réponses apportées demeurent à leurs yeux insuffisantes. Certaines de ces revendications trouveront toutefois un écho auprès de la Commission européenne, qui promet un plan d'action communautaire pour accroître les ressources humaines dans le secteur scientifique, et en particulier dans le secteur public et universitaire.

LES UNIVERSITÉS OCCUPENT UNE PLACE CENTRALE dans le système allemand de recherche et d'innovation, et entendent être partie prenante des efforts nationaux d'amélioration des performances et de l'attractivité de l'activité scientifique. Cette politique s'inscrit bien évidemment dans la perspective des engagements communautaires pour une Europe de la connaissance et la constitution de l'Espace Européen de la Recherche. Cela implique des efforts soutenus en termes de constitution de réseaux, d'interdisciplinarité face à la complexité des problèmes scientifiques et sociaux à résoudre, et de recherche de l'excellence. A cet égard, les établissements allemands semblent exploiter au mieux les outils que leur offrent les programmes européens, et en premier lieu les PCRDT. Mais la réputation d'excellence ne se décrète pas. Elle s'acquiert au fil du temps et nécessite pour cela une certaine stabilité et visibilité des moyens financiers et humains mis à disposition pour le système dans son ensemble, et non seulement pour quelques établissements.

\section{Indications bibliographiques}

BUNDESMINISTERIUM FÜR BILDUNG UND FORSCHUNG (BMBF), Faktenbericht Forschung 2002, Bonn, 2002 (www.bmbf.de)

HOCHSCHULREKTORENKONFERENZ (HRK), «Zur aktuellen hochschulpolitischen Diskussion ». Erklärung des 98. Senats der Hochschulrektorenkonferenz vom 10.2.2004 (www.hrk.de)

COMMISSION DES COMMUNAUTÉS EUROPÉENNES, Le rôle des universités dans l'Euroupe de la Connaissance, Communication de la Commission $\operatorname{COM(2003)} 58$ final, Bruxelles, 0502-2003 (www.europa.eu.int)

Kowi-aktuell n53, 21-03-2003 (www.kowi.de)

DFG, FHG, LeIBNIZ-GemeINSCHAFT, HGF, HRK, MPG, WR, Wachstum braucht Wissenschaft : Bildung und Forschung bilden Basis und Motor wirtschaftlicher und sozialer Innovation, 12-2-2004 (www.hrk.de)

OBSERVATOIRE DES SCIENCES ET DES TECHNIQUES, Rapport préliminaire des participations françaises au cinquième Programme Cadre, septembre 2003 (www.eurosfaire.prd.fr) 